\title{
Large Aperture Metal-Mesh Lenses for THz Astronomy
}

\author{
Paul Moseley ${ }^{1}$, Giorgio Savini ${ }^{2}$, and Peter Ade ${ }^{1}$ \\ ${ }^{1}$ School of Physics and Astronomy, Cardiff University, the Parade, CF24 3AA Cardiff, Wales, UK \\ ${ }^{2}$ Optical Science Laboratory, Physics and Astronomy Department, University College London,
} Gower Street, London WC1E 6BT, UK

\begin{abstract}
In this paper we present preliminary results of a metal-mesh graded index Fresnel lens. The designed lens has a diameter of $300 \mathrm{~mm}$, a focal length of $1050 \mathrm{~mm}$ and operates from 70 to $300 \mathrm{GHz}$. It was not possible to directly scale the design of the previous lenses, so a Fresnel graded index was used. A new testing facility was developed and verified, which allowed us to show that the new lens matches the same performance of a traditional polyethylene lens. Lenses of this diameter are of interest in the astronomy instrumentation community.
\end{abstract}

Index Terms-GRIN Lens, Metamaterials, THz Astronomy.

\section{INTRODUCTION}

There have been previous demonstrations that a metamaterial lens can be manufactured to match the performance of a normal polyethylene (PE) lens [1,2]. These demonstrated lenses were approximately $70 \mathrm{~mm}$ in diameter, while there are some applications where lenses of that size are required, there is a greater demand for larger diameter devices in the astronomy community. One such application is in the next generation of $\mathrm{CMB}$ telescopes which require large fore optics capable of operating over $70-300 \mathrm{GHz}$ [3]. Previous designs have either used all reflective optics or used refractive optics consisting of polyethylene or silicone lenses.

Due to the size and bulk of the lenses required, manufacturing and applying suitable antireflection coatings is difficult, which makes them a less favorable option. A metamaterial flat lens offers an advantage over these lenses due to the fact that they are more compact and can be made and coated in one process. In this paper we present the design and preliminary results of a $300 \mathrm{~mm}$ diameter Fresnel metamaterial lens.

\section{LENS DESIGN}

To design a large aperture metamaterial lens using the same approach as the previous lens [1], which was based on the Woods lens formalism [5], is not straight forward. Scaling the original $70 \mathrm{~mm} \mathrm{~F} 3.5$ lens up to $300 \mathrm{~mm}$ in diameter requires increasing either the central refractive index, the overall thickness (greater number of mesh layers) or a combination of both. Given that it is not possible to get a refractive index below the index of the substrate (1.5) with broadband capacitive meshes, the centre refractive index has to increase to over 5 . This is possible using a metal mesh structure that would cover the frequency range of interest, but, the increase in thickness required means that over 60 mesh grids are required which is not practical to build.

An alternative approach to address this issue is to design a lens based on a Fresnel graded index profile. This is designed in a similar way to a traditional Fresnel lens, replacing concentric rings of increasing curvature, with increased variation of the index gradient. This allows for the overall index range to be compressed, so that required index values can be scaled to a more favorable region. Figure 1 shows the refractive index profile of a $300 \mathrm{~mm}$ Fresnel lens with a focal length of $1050 \mathrm{~mm}$, optimized for $150 \mathrm{GHz}$. The variation in index is achieved by applying a radial profile to the size of square copper patches [6], then 20 of these identical mesh layers are combined, separated by $100 \mu \mathrm{m}$ polypropylene, yielding a total thickness of $2 \mathrm{~mm}$. The centre index of 2.7 was chosen so that the previous metamaterial parameter space from the last lens can be used, thus limiting the number of free parameters.

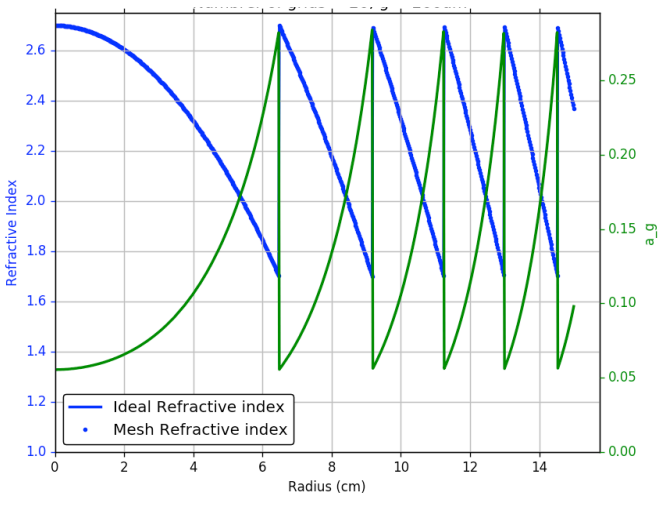

Figure 1. The refractive index distribution of the Fresnel lens (blue curve) and the corresponding mesh parameters (green curve).

The lens is fabricated using a hot press technique [7] to produce a solid robust device which is shown in Figure 2. 


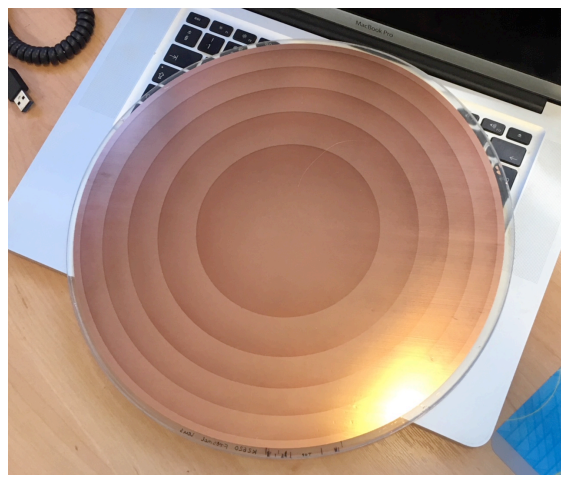

Figure 2. The completed 300mm metal mesh Fresnel lens.

\section{TeSt SETUP}

The lens presented here is considerably larger than any previous lenses tested. Therefore, a new experimental test setup had to be created. The initial test configuration was setup to scan through the focus in three dimensions to gauge the properties of the lens. The lens was placed in a collimated beam that fully illuminates the lens surface. The setup design for this is shown in Figures Figure 3\&Figure 4. A segment of a $2 \mathrm{~m}$ spherical mirror with focal length $2 \mathrm{~m}$ was used. The transmitting VNA frequency extender was placed at the mirror focus such that the reflected beam off the mirror is collimated. Alignment checks were performed that show when a laser scanned across the collimated beam the laser spot always focused at the same point on the transmitter. The receiver was mounted on XYZ stage which gives submillimeter positioning capability. The entire system including the data acquisition is controlled by LabVIEW.

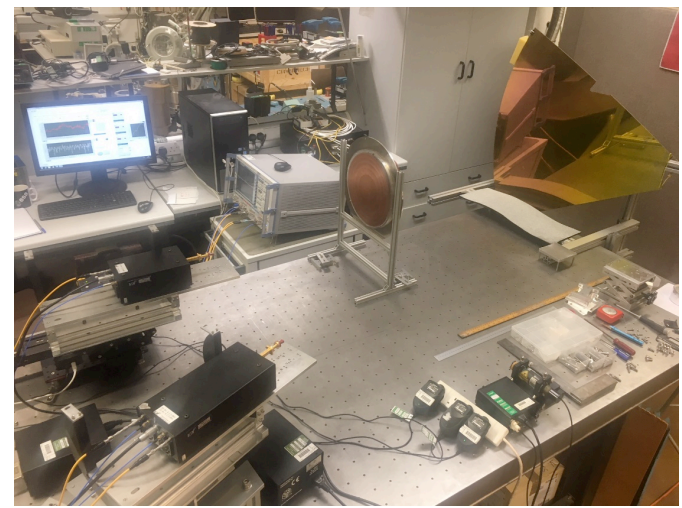

Figure 3. The test setup used for measuring the lens. Transmitter is at the bottom of the image.

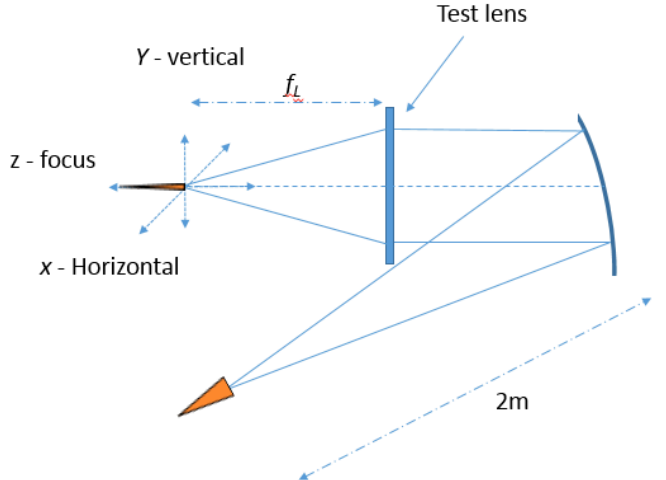

Figure 4. Schematic of the test setup. The position of the lens holder was chosen to limit any vignetting of the beam coming from the transmitter.

\section{A. RF Validation}

To verify the performance of the test setup, a series of measurements were performed which are shown in Figure 5. Firstly, the clear aperture of the lens holder is scanned (blue curve) to ensure that there is uniform illumination. Secondly, the same scan is performed with the aperture blocked (red curve) to ensure that there is no significant stray light entering the receiver. Finally, a known polyethylene lens was characterized (yellow curve). It can be seen that there is good uniform illumination across the surface of the lens and minimal stray light.

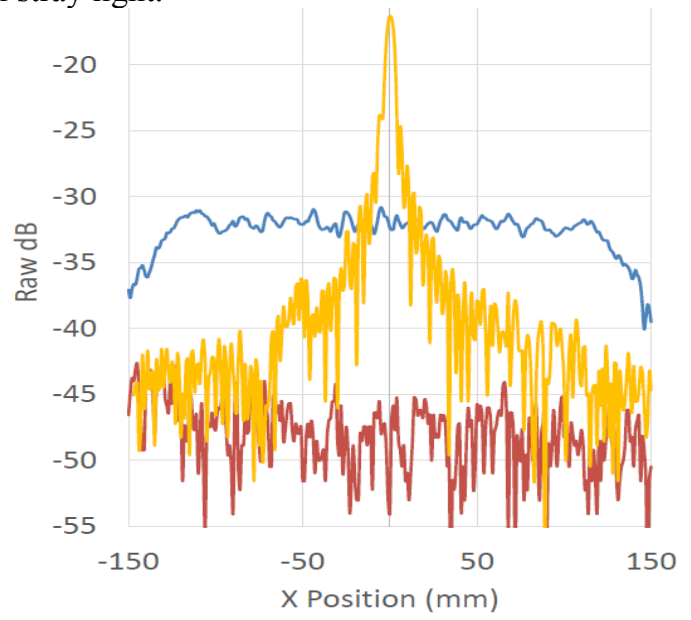

Figure 5. Initial RF verification beam scans in $x$. Open aperture (blue), obscured aperture (red) and PE lens (yellow). Plots are in raw dB all taken with the same VNA output power.

\section{PRELIMINARY LENS RESULTS}

A series of initial beam scans of the Fresnel lens were performed at two frequency bands, $75-110 \mathrm{GHz}$ and $160-$ $260 \mathrm{GHz}$. The results of the beam cuts taken at the focus of the lens at 108 and $233 \mathrm{GHz}$ are shown in Figures $5 \& 6$. For comparison the predicted airy function of an ideal lens with corresponding size and focal length is over plotted, both frequencies show good agreement with the model. 


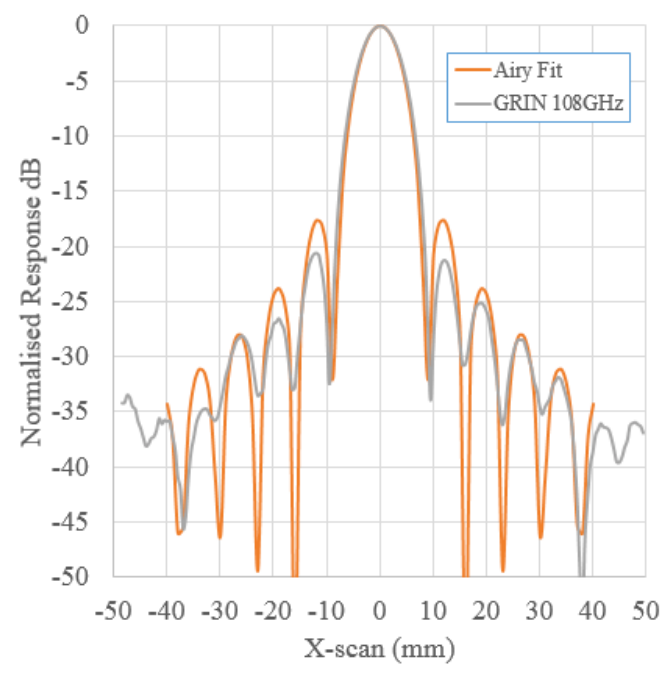

Figure 6. Beam cut at the focus of the Fresnel lens at 108GHz (Grey) compared to predicted airy model (Orange).

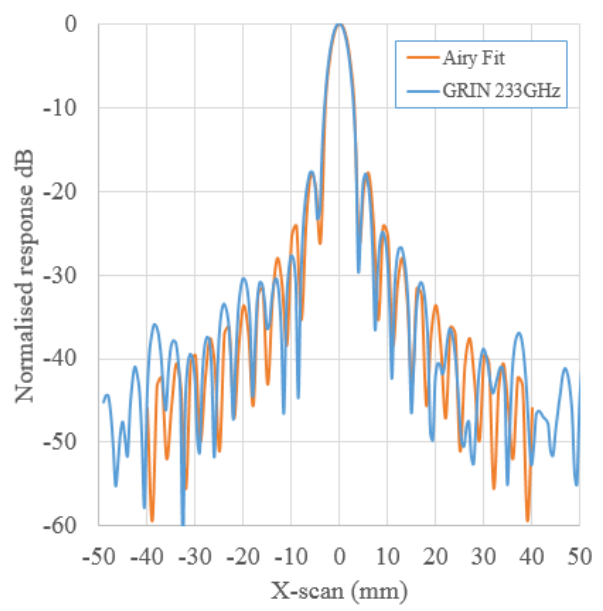

Figure 7. Beam cut at the focus of the Fresnel lens at $108 \mathrm{GHz}$ (Blue) compared to predicted airy model (Orange).

\section{CONCLUSION}

We have presented initial results showing that the focusing properties of a large metamaterial lens matches that of an ideal lens. In a full paper we will present further results detailing the precise chromatic behavior, cross polarisation levels and insertion loss of the lens.

\section{REFERENCES}

[1] Savini, G., Ade, P. A., \& Zhang, J. (2012). A new artificial material approach for flat $\mathrm{THz}$ frequency lenses. Optics express, 20(23), 25766-25773.

[2] Pisano, G., Ng, M. W., Haynes, V., \& Maffei, B. (2012). A broadband metal-mesh half-wave plate for millimetre wave linear polarisation rotation. Progress In Electromagnetics Research M, 25, 101-114.
[3] Abitbol, M. H., Ahmed, Z., Barron, D., Thakur, R. B., Bender, A. N., Benson, B. A., ... \& Chuss, D. T. (2017). CMB-S4 Technology Book. arXiv preprint arXiv:1706.02464.

[4] Merchand, E. (2012). Gradient index optics. Elsevier.

[5] M. Young, The Technical Writer's Handbook. Mill Valley, CA: University Science, 1989

[6] Zhang, J., Ade, P. A., Mauskopf, P., Moncelsi, L., Savini, G., \& Whitehouse, N. (2009). New artificial dielectric metamaterial and its application as a terahertz antireflection coating. Applied optics, 48(35), 6635-6642.

[7] Ade, P. A., Pisano, G., Tucker, C., \& Weaver, S. (2006, July). A review of metal mesh filters. In Proc. SPIE (Vol. 6275, p. 62750U). 